\title{
Accelerated endurance test of single-mode vertical-cavity surface- emitting lasers under vacuum used for a scalar space magnetometer
}

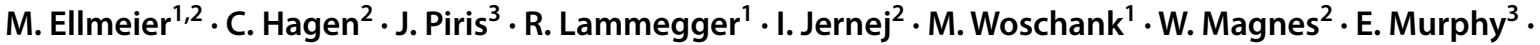 \\ A. Pollinger ${ }^{2} \cdot$ C. Erd $^{4} \cdot$ W. Baumjohann ${ }^{2} \cdot$ L. Windholz ${ }^{1}$
}

Received: 28 July 2017 / Accepted: 23 December 2017 / Published online: 6 January 2018

(c) The Author(s) 2017. This article is an open access publication

\begin{abstract}
We performed an endurance test with single-mode vertical-cavity surface-emitting lasers (VCSEL) under vacuum condition and increased operational parameters (laser current and laser temperature) to accelerate the aging of the lasers. During the endurance test the emitted polarization-dependent and polarization-independent optical light power from the lasers was detected. Additionally, electro-optical characterisations including measurements of the combination of laser current and laser temperature to excite the ${ }^{87} \mathrm{Rb} \mathrm{D}_{1}$ transition $(\lambda=795 \mathrm{~nm})$, the current and temperature tuning coefficients, laser line width, threshold current and the polarization ellipse were performed for the aged lasers. The test was started with a number of 12 VCSELs consisting of 4 lasers each from 3 different suppliers. The aging behaviour of VCSELs was investigated with respect to the development of a new optical magnetometer prototype for space missions with a mission duration of up to 17 years. Only a limited change of the electro-optical parameters can be tolerated by the instrument design over the mission duration. The endurance test and the electro-optical characterizations revealed clear differences in the aging behaviour of the three suppliers. Lasers from one supplier showed that they can be operated for more than 17 years under vacuum conditions without major degradation of their operational parameters.
\end{abstract}

\section{Introduction}

In the last two decades, laser diodes have become important in experimental science. Especially vertical-cavity surfaceemitting lasers (VCSEL) have a versatile field of application. Compared to other laser diodes VCSELs have low production costs, high modulation efficiency and their circular beam profile allows simple fibre coupling [1]. Therefore, they are widely used in optical fibre communication, in spectroscopy [2] and in consumer electronics.

VCSELs are also interesting for space applications such as for data communications inside of satellites or new

M. Ellmeier

michaela.ellmeier@tugraz.at

1 Institute of Experimental Physics, Graz University of Technology, Graz, Austria

2 Space Research Institute, Austrian Academy of Sciences, Graz, Austria

3 Directorate of Technology, Engineering and Quality, European Space Agency, Noordwijk, The Netherlands

4 Future Missions Department, Directorate of Science, European Space Agency, Noordwijk, The Netherlands scientific instrument developments [3]. For this application, it has to be demonstrated that VCSELs can tolerate the harsh conditions in space and also provide the required lifetime of several years for space missions.

In this paper we summarize the results of a long-term endurance test under vacuum condition which was accomplished at the Optics- and Opto-Electronics Laboratory (OOEL) [4] of the European Science and Technology Centre (ESTEC) with VCSELs from three different manufacturers. The tested laser diodes were all single-mode VCSELs emitting at a wavelength of $795 \mathrm{~nm}$ hermetically sealed in a TO 46 package. The test was carried out under vacuum condition to enable testing as close as possible to the environmental conditions in space. For example, leakages in the sealing of the lasers could occur which would cause a loss of the package atmosphere and thus could have an influence on the lifetime [5].

This test was carried out in the context of the development of a new space magnetometer prototype, the Coupled Dark State Magnetometer (CDSM) [3, 6]. The CDSM is a type of coherently optically pumped magnetometer. Its functional principle is based on the measurement of the Zeeman energy level shift of the ground state within 
the Zeeman manifold of the ${ }^{87} \mathrm{Rb} \mathrm{D}_{1}$ hyperfine structure caused by an external magnetic field. This energy level shift is measured using a quantum interference effect called Coherent Population Trapping (CPT) [7, 8]. From this shift, the external magnetic field can be calculated by means of the Breit-Rabi formula [9].

A prototype has been developed by the Institute of Experimental Physics of the Graz University of Technology (TUG) and the Space Research Institute (IWF) of the Austrian Academy of Sciences.

The first demonstration of this new magnetometer in space will take place aboard the Chinese Seismo-Electromagnetic Satellite (CSES) mission in spring 2018.

The CDSM was also selected for the JUpiter ICy Moon Explorer (JUICE) mission of the European Space Agency (ESA) which will be launched in 2022. This mission will visit the Jovian system and will investigate, among others, the magnetic field around Jupiter's moon Ganymede, which will help studying its inner structure including a presumed subsurface ocean.

For the development of the CDSM, VCSELs are used because their properties fit the requirements for the preparation of the CPT effect and also fulfil those for the use in space applications such as small size, low power consumption and robustness. For the excitation of the CPT effect we use a single-mode VCSEL emitting a carrier wavelength of $\lambda=794.979 \mathrm{~nm}$ (vacuum wavelength) [10]. On this carrier frequency we apply a modulation frequency of $3.417 \mathrm{GHz}$ via the power supply current of the VCSEL, which corresponds to the half of the ground-state splitting frequency of ${ }^{87} \mathrm{Rb}$.

Therefore, one of the biggest advantages of the VCSEL for our application is its high modulation ability of several gigahertz. However, a disadvantage of this laser type for the use in CPT resonance excitation applications can be its typically higher laser line width of about $50-100 \mathrm{MHz}$ compared to, e.g. DFB lasers (2-5 MHz). The laser line width should be less or equal to the homogenous line width of the excited optical resonance to allow an efficient preparation of the CPT effect [11,12]. A further drawback is its high current tuning coefficient (see Table 5). Compared to edge emitters this coefficient is about a factor of ten higher. Thus, current noise stemming from the laser's constant current source is more efficiently translated into frequency noise leading to a higher noise level in the magnetic field measurement.

For the JUICE mission it is important to prove that the VCSELs can operate for several years (up to 17 years) under vacuum condition and retain their optical properties required for a proper function of the CDSM. Therefore, the VCSELs were artificially aged under vacuum condition to investigate their lifetime and repeatedly electro-optically characterised in the course of the test [13].
In Sect. 2 a short summary of VCSEL's reliability testing is given. Sections 3 and 4 summarize the test setup and the most important results.

\section{VCSEL reliability and test parameters}

In general, there are several reasons for failure of VCSEL diodes. Commonly, they are divided into failures which cause the diodes to malfunction suddenly and those that cause a gradual slow deterioration of the laser properties like optical power [14], which is called wear-out.

Abrupt failure is caused for example by growth of dislocation networks often described as dark line defects due to material defects, Electro Static Discharge (ESD) or mechanic damage $[15,16]$.

In general, the lifetime of a VCSEL significantly depends on its parameters of operation [17]. High junction temperatures and high current densities-especially in the active region-reduce the lifetime of a VCSEL. Thus, by increasing the VCSEL's current and temperature compared to their typical operational values $I_{\mathrm{op}}$ and $T_{\mathrm{op}}$, the lasers aging can be accelerated and the VCSELs' behaviour of several years of operation can be studied within a much shorter time. Depending on the chosen test parameters and the design of the VCSEL the lasers are aged at different rates. The factor by which the aging process is increased is called acceleration factor AF. It is defined as the ratio of the time-to-failure at nominal operational conditions $\mathrm{TTF}_{\mathrm{op}}$ to that at accelerated operational conditions $\mathrm{TTF}_{\text {test }}$.

The acceleration factor reached for the VCSELs is calculated according to following formula [17]:

$$
\mathrm{AF}=\frac{\mathrm{TTF}_{\mathrm{op}}}{\mathrm{TTF}_{\text {test }}}=\left(\frac{I_{\text {test }}}{I_{\mathrm{op}}}\right)^{n} \exp \left(\frac{E_{\mathrm{A}}}{k_{\mathrm{B}}}\left(\frac{1}{\mathrm{Tj}_{\mathrm{op}}}-\frac{1}{\mathrm{Tj}_{\text {test }}}\right)\right)
$$

Here $I_{\text {test }}$ is the applied laser current during the endurance test and $I_{\mathrm{op}}$ the typical (nominal) operational current value in our magnetometer application. The exponent $n$ as well as the activation energy $E_{\mathrm{A}}$ are empirically determined by the manufacturer during their reliability testing. $k_{\mathrm{B}}$ is the Boltzmann constant, $\mathrm{Tj}_{\mathrm{op}}$ is the junction temperature for nominal operation and $\mathrm{Tj}_{\text {test }}$ the junction temperature for the test temperature. However, the junction temperature of the VCSEL cannot be measured directly and is calculated via the relation [1]:

$\mathrm{Tj}_{x}=T_{x}+R_{\mathrm{th}} \cdot\left(I_{x} \cdot V_{x}-P_{x}\right)$

The junction temperature depends on the ambient temperature $T_{\mathrm{x}}$ and the heating of the laser chip by electrical power dissipation during its use. The heat that is generated by the laser itself is calculated with the thermal resistance $R_{\mathrm{th}}$ and the difference between the introduced electrical power (laser 
current $I_{\mathrm{x}}$ times laser voltage drop $V_{\mathrm{x}}$ ) and the emitted optical power $P_{\mathrm{x}}$. For the calculation of the acceleration factor the parameters $n, E_{\mathrm{A}}$ and $R_{\mathrm{th}}$ were provided by the VCSEL manufacturers [13].

The nominal operational parameters $\left(I_{\mathrm{op}}\right.$ and $\left.T_{\mathrm{op}}\right)$ of each VCSEL were determined during the first electro-optical characterisation. The choice of the operational current was based on the required optical power $P_{\mathrm{x}}$ for the operation of the magnetometer. The corresponding laser temperature $T_{\mathrm{op}}$ was determined to achieve a wavelength of $\lambda=794.979 \mathrm{~nm}$ and varies for each laser which influences the obtained equivalent operational life time $\tau_{\text {eop }}$ (see Table 2).

The test was planned with four VCSELs at a time from three different suppliers. The abbreviations for the different suppliers which will be used further on and the corresponding part numbers as well as the operational currents $I_{\mathrm{op}}$ and the applied current during the endurance test $I_{\text {test }}$ are stated in Table 1.

The test duration was first 1 year and then extended further for 2 months. The ambient temperature $T_{\mathrm{x}}$ during the vacuum test was set to $70^{\circ} \mathrm{C}$, which was the highest possible temperature of the test setup. The applied laser current $I_{\text {test }}$

Table 1 Part numbers, operational laser currents $I_{\text {op }}$ as well as laser current during the endurance test $I_{\text {test }}$ for the used lasers

\begin{tabular}{llll}
\hline Supplier & Part number & $I_{\text {op }}(\mathrm{mA})$ & $I_{\text {test }}(\mathrm{mA})$ \\
\hline A & VIX-795S-0000-G002 & 1.9 & 1.9 \\
B & V795-2222-001 & 2.9 & 3.0 \\
C & APM2101013300 & 3.5 & 4.0 \\
\hline
\end{tabular}

for VCSELs from supplier B and C was set to their maximum rating according to the data sheet. The reached equivalent operational life time $\tau_{\text {eop }}$ of each tested VCSEL and the mean acceleration factor $\mathrm{AF}_{\text {mean }}$ can be found in Table 2.

\section{Laser endurance test description}

The main goals of the laser endurance test were to investigate the aging behaviour of VCSELs under vacuum condition and to determine if the optical and electrical properties of the laser degrade over the achieved lifetime. All used VCSELs are single-mode lasers emitting at $795 \mathrm{~nm}$ which are hermetically sealed in a TO 46 package.

Before the lifetime test 12 VCSELs in total (4 each from supplier A, B and C) were selected and electro-optically characterized. For the vacuum test, the selected lasers were mounted in two separate vacuum chambers at the Opticsand Opto-Electronics Laboratory (OOEL) of the European Science and Technology Center (ESTEC) [4]. Half of the lasers ( 2 from each supplier) were mounted in one vacuum chamber (see Fig. 1) where they remained permanently for the whole vacuum test duration, while the lasers in the second chamber were removed twice for electro-optical characterization.

Two VCSELs each were assembled in mounting blocks which contained two biconvex lenses to form two collimated Gaussian laser beams and a Pt1000 element to measure the temperature of the mounting blocks individually. The mounting blocks were fixed inside the vacuum chamber on temperature-stabilized carriers. The mean temperature

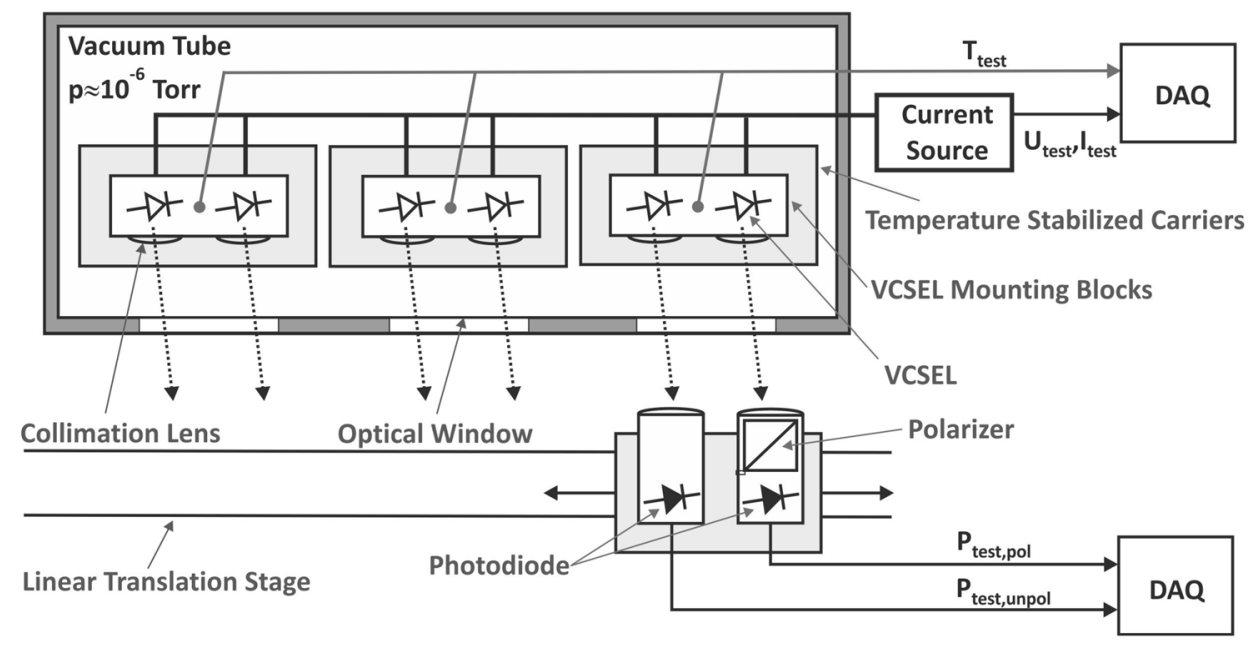

Fig. 1 Optical and electrical setup of the endurance test under vacuum condition: the lasers were mounted in mounting blocks on temperature-stabilized carriers. The mounting blocks contained lenses for the collimation of the laser beams and a Pt1000 element to measure the temperature. Once per hour the temperature $T_{\text {test }}$, current $I_{\text {test }}$ and voltage $U_{\text {test }}$ of the lasers as well as the emitted optical power were measured. The polarization-independent $P_{\text {test,unpol }}$ and the polarization-dependent optical power $P_{\text {test,pol }}$ were detected outside the vacuum chamber by two photodiodes and one additional polarizer. Both photodiodes were moved between the windows by a linear translation stage 
during the test was $(65.6 \pm 2.0){ }^{\circ} \mathrm{C}$ in vacuum chamber 1 and $(66.1 \pm 1.8){ }^{\circ} \mathrm{C}$ in vacuum chamber 2 . Each VCSEL was supplied with its own custom-made current source providing the predefined currents (see Sect. 2).

The vacuum chambers have several optical windows which allowed the detection of the optical power outside of the chambers via photo diodes. One photo diode measured the total laser power while the other photo diode detected the laser polarization-dependent optical power with the help of an additional linear polarizer placed in front of the photo diode. Thus, changes in the orientation of the polarisation plane like the unwanted arbitrary polarization jumps could be measured. All VCSEL were mounted in such a way that the maximum of the linear polarization state was measured. For these measurements, the two optical detectors were moved from one optical window to the next by a linear translator.

Over the whole test duration the laser current $I_{\text {test }}$, laser voltage $U_{\text {test }}$, temperature $T_{\text {test }}$, polarization-dependent laser power $P_{\text {test,pol }}$ and polarization-independent laser power $P_{\text {test,unpol }}$ were measured and logged by a data acquisition system once per hour. The recorded laser power of each laser as a function of test duration is presented in Sect. 4.1.

For all lasers, the selected electrical and optical parameters were measured before and after the accelerated aging test (preliminary and final electro-optical characterization). Additionally, VCSELs mounted in the vacuum chamber number 1 were characterized twice during the accelerated aging test [intermediate characterization (IC)]. These characterization measurements were performed outside the vacuum chamber on an optical bench. Therefore, the VCELSs were dismounted from the vacuum chamber mounting blocks and assembled into a laser setup which allowed changing and stabilizing both the laser temperature (within $\pm 1 \mathrm{mK}$ ) and laser current. The laser temperature was measured with a Pt1000 element using a four-wire sensing schema and was set with the help of a thermoelectric cooler driven by a temperature control unit (feedback loop). The laser current could be changed by an adjustable constant current source and was monitored with a precision multimeter to an accuracy of $\pm 10 \mathrm{nA}$.

The following measurements were performed during the electro-optical characterisation: laser current and temperature combinations for achieving a constant wavelength of $\lambda=794.979 \mathrm{~nm}$ (vacuum wavelength), current tuning coefficient, temperature tuning coefficient, laser line width, threshold current and polarization ellipse.

The determination of the emitted laser frequency was calibrated by measuring the resonant excitation inside a glass cell filled with isotopically pure ${ }^{87} \mathrm{Rb}$. To obtain resonant excitation of the rubidium atoms, a sawtoothshaped modulation was applied onto the laser current to sweep the laser frequency. During the sweep the transmitted laser light through the cell was measured by a photo diode. The detected photo diode signal was depicted with an oscilloscope showing the ${ }^{87} \mathrm{Rb} \mathrm{D}_{1}$ spectrum. The ${ }^{87} \mathrm{Rb}$ $\left(5^{2} \mathrm{~S}_{1 / 2} F=2 \rightarrow 5^{2} \mathrm{P}_{1 / 2} F=1\right)$ transition was used as reference to set the laser frequency. At certain laser current values within the operational range of the laser, the laser temperature was adapted such that this selected transition was excited and the corresponding laser current and temperature values were read.

At the same measured laser current-temperature pairs, also the current tuning coefficient $\Delta \nu / \Delta I$ and the temperature tuning coefficient $\Delta \nu / \Delta T$ were determined. For the measurement of the current tuning coefficient the laser temperature was fixed and the difference of the laser current $\Delta I$ required to change the laser frequency from the ${ }^{87} \mathrm{Rb}\left(5^{2} \mathrm{~S}_{1 / 2} F=2 \rightarrow 5^{2} \mathrm{P}_{1 / 2} F=1\right)$ transition to the ${ }^{87} \mathrm{Rb}$ $\left(5^{2} \mathrm{~S}_{1 / 2} F=1 \rightarrow 5^{2} \mathrm{P}_{1 / 2} F=2\right)$ transition measured. With the known frequency spacing $(\Delta \nu=7.650 \mathrm{GHz})$ between these two transitions, the current tuning coefficient $\Delta \nu / \Delta I$ was calculated.

For the measurement of the temperature tuning coefficient $\Delta \nu / \Delta T$ a similar approach was used. Now, the laser current was fixed and the difference in laser temperature required to change the laser frequency between these two transitions was measured. Again from the known frequency spacing $\Delta \nu$ the temperature tuning coefficient $\Delta \nu /$ $\Delta T$ was calculated.

The laser line width was measured with the help of an optical spectrum analyser. The used device has a free spectral range of $\delta_{\mathrm{FSR}}=1980 \mathrm{MHz}$, finesse of $F \approx 300$ and thus a resolution of approximately $\delta \nu=7 \mathrm{MHz}$. A measurement uncertainty of about $\pm 5 \mathrm{MHz}$ could be obtained for the laser line width.

The threshold current was determined by making use of the appearance of the speckle pattern when increasing the laser current from zero to above the threshold current. If the laser is operated at a laser current below the threshold current, the laser diode emits virtually incoherent light. At the threshold current the laser starts emitting coherent light and a speckle pattern becomes visible on a rough surface. With the help of an infrared sensitive camera the appearance of the pattern was determined by slowly increasing the laser current from zero up to the threshold current. With this method the threshold current can be determined with an accuracy of $\pm 0.1 \mathrm{~mA}$.

The polarization ellipse of the linear polarisation state of the laser light was also investigated. The maximum and the minimum of the ellipse were determined with the help of a Glan-Thompson polarizer and a power meter.

The obtained results of the electro-optical tests are presented in Sect. 4.2. 


\section{Test results}

\subsection{Long-term behaviour of optical laser power under vacuum condition and accelerated aging}

In total the VCSELs in chamber 1 were operated for 344 days and the VCSELs in chamber 2 for 414 days. For the lasers in vacuum chamber 1 , the first intermediate characterisation (1st IC) took place after 114 days and the second one (2nd IC) was carried out after 288 days. Chamber 2 was not opened during the entire test duration.

During the test, all lasers in both chambers were switched off for maintenance for 1 week each (chamber 1 after 114 and 275 days, chamber 2 after 147 and 308 days).

For the illustration of the measurement results in Fig. 2, the measured data were smoothed using a moving average over 20 readings because the raw data were noisy due to the applied measurement method with the linear translator (see Sect. 3). This artificial noise resulted from slight variations in the position of the detectors and was not caused by the VCSELs itself.

The lasers were labelled with their respective supplier letter (A, B or C) and additionally the two lasers from each supplier which were mounted in vacuum chamber 1 got the numbers 1 and 2 and the two further lasers from the same supplier installed in vacuum chamber 2 received the numbers 3 and 4 . Further, the label of the initially mounted lasers ends with a number 1 and the replaced lasers with a number 2. For example, laser number 2 from supplier A which was initially mounted in vacuum chamber 1 was named A2.1 and was replaced by laser A2.2. Figure 2 includes both the polarization-independent and polarization-dependent laser power, which is marked with an additional suffix $\mathrm{p}$.

The laser failure criterion was defined as the reduction of the measured laser power below $50 \%$ of its initial value $P_{\mathrm{I}}$ at $T_{\text {test }}$. This power reduction can be tolerated by the setup and allows a proper operation of the magnetometer.
Fig. 2 Behaviour of the optical power of all tested lasers during the accelerated endurance test under vacuum condition over the test duration $\Delta t_{\text {test }}:$ the left column contains the results from vacuum chamber 1 and the right column from vacuum chamber 2. Figures $\mathbf{a}$ and $\mathbf{b}$ show the long-term behaviour of the optical power of lasers from supplier A, $\mathbf{c}$ and $\mathbf{d}$ from supplier B and $\mathbf{e}$ and $\mathbf{f}$ from supplier $\mathrm{C}$
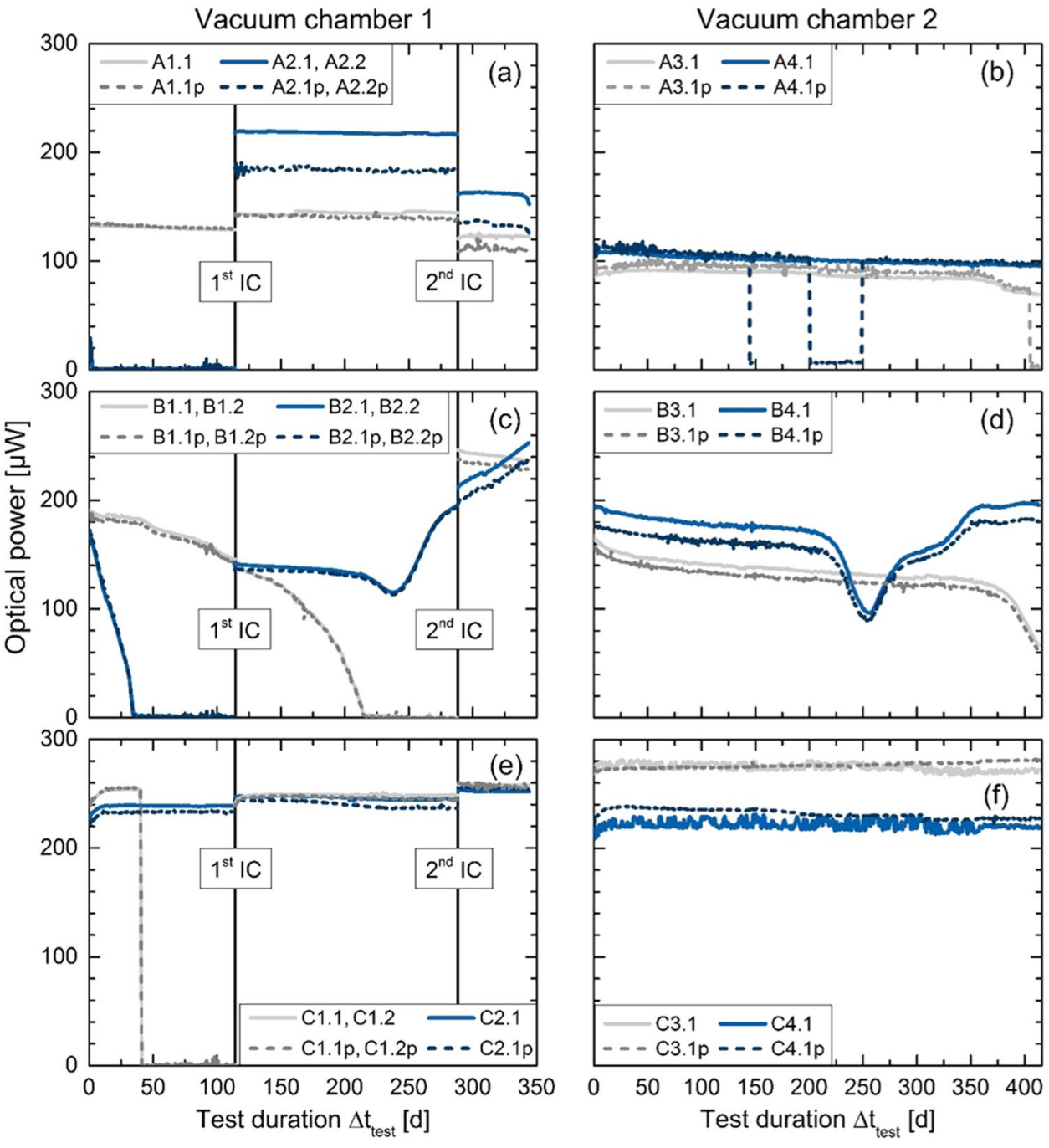
All lasers showed an initial reduction of their optical power when the laser temperature was increased to $T_{\text {test }}$ and the vacuum established in the chambers. Within the first few hours the laser power increased again and stabilized. As initial value the optical power after this transient behaviour was taken.

In the course of the intermediate characterisations the functioning lasers were optically and electrically measured and the failed lasers replaced.

The discontinuities of the optical power after the intermediate characterizations in Fig. 2 resulted from the complete dismounting of the lasers from the mounting blocks and a slightly different alignment after reassembly. Due to this discontinuities, the relative changes in optical power after the intermediate characterization were related to the new starting value after the intermediate characterization.

In Fig. 2a and $b$ the results of the vacuum test for supplier A are summarized. Laser A1.1 showed only minor relative changes of its optical power (about $3 \%$ ) during the test phases. In contrast the optical power of laser A2.1 started degrading immediately after mounting in the vacuum chamber and failed on the second day of operation in vacuum. Laser A2.1 was replaced during the 1st IC by laser A2.2. The optical power of laser A2.2 behaved quite stable until the last test month where its optical power reduced by about $5 \%$.

Also the laser power of A3.1 only showed reduction of its optical power by about 3\% until the 350th day of operation in the vacuum test. Then the laser power decreased faster until the 405th day (in total about 20\%) and revealed a polarization jump. The polarization jump is visible as a leap in the laser polarization-dependent optical power and no change in the polarization-independent power. The polarisation of laser A4.1 revealed in total two polarisation jumps within the whole test period. The first polarization jump appeared shortly before the first switch off for maintenance after 144 days of operation. After switching on the lasers again the original polarization plane was restored. The second polarization jump appeared after 200 days and returned to its initial polarization plane on the 249th day (see Fig. 3). The change of the polarization state in Fig. 3 took up to $20 \mathrm{~h}$ as well as the return to the original polarization state. The change of the polarization state of laser A3.1 required as well $20 \mathrm{~h}$. On the contrary, the first polarization jump of laser A4.1 took only $3 \mathrm{~h}$.

In total, laser A4.1 exhibited a gradual decrease of its optical power by about $9 \%$ by the end of the test.

Figure $2 \mathrm{c}$ and $\mathrm{d}$ show the long-term behaviour of the optical power under vacuum condition for supplier B. Laser B2.1 started degrading right from the test start and failed after 21 days of operation in the vacuum chamber. It was replaced by laser B2.2 which showed an unusual reduction of its optical power $\left(-16 \%\right.$ of initial power $\left.P_{\mathrm{I}}=142 \mu \mathrm{W}\right)$ with a subsequent increase $(+34 \%)$ until the 2 nd IC. After the 2 nd IC

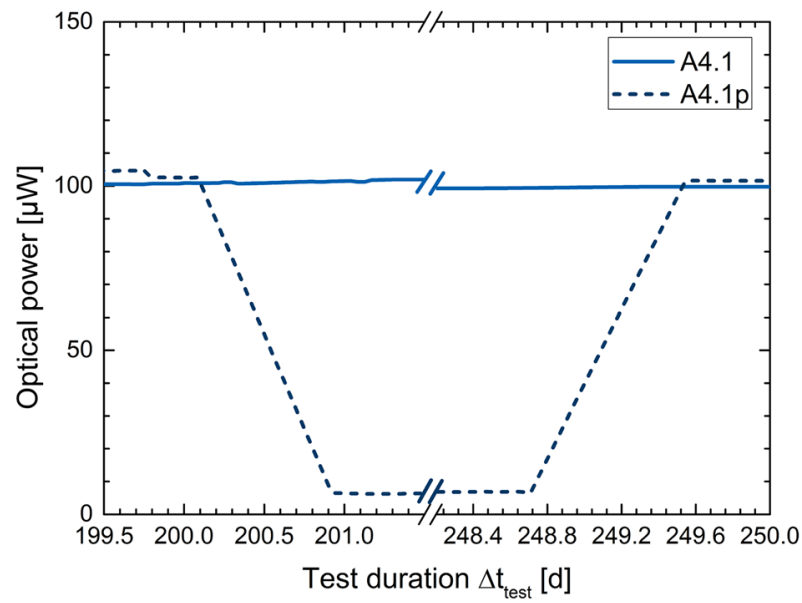

Fig. 3 Polarization jump detected in the run of the optical power of laser A4.1: the polarization-dependent power was reduced but the polarization-independent power was unchanged. After 49 days the original polarization state was restored

Table 2 Calculated maximum equivalent operational lifetime $\tau_{\text {eop }}$ for all tested lasers at the end of the accelerated aging test: the factor $\mathrm{AF}_{\text {mean }}$ is mainly influenced by the different operational temperatures $T_{\text {op }}$ of each laser

\begin{tabular}{llccc}
\hline Laser & $T_{\text {op }}\left({ }^{\circ} \mathrm{C}\right)$ & $\Delta t_{\text {test }}$ (days $)$ & $\mathrm{AF}_{\text {mean }}$ & Max. $\tau_{\text {eop }}(\mathrm{a})$ \\
\hline A1.1 & 24.2 & 344 & 21.7 & 20.5 \\
A2.1 & 24.2 & $2^{\mathrm{a}}$ & 21.6 & 0.1 \\
$\mathrm{~A} 3.1$ & 26.5 & 414 & 17.5 & 19.9 \\
$\mathrm{~A} 4.1$ & 27.3 & 414 & 16.3 & 18.5 \\
$\mathrm{~A} 2.2$ & 22.3 & 230 & 20.7 & 13.1 \\
$\mathrm{~B} 1.1$ & 19.2 & $174^{\mathrm{a}}$ & 7.1 & 3.4 \\
$\mathrm{~B} 2.1$ & 21.6 & $21^{\mathrm{a}}$ & 5.0 & 0.3 \\
$\mathrm{~B} 3.1$ & 21.3 & $403^{\mathrm{a}}$ & 6.5 & 7.2 \\
$\mathrm{~B} 4.1$ & 22.3 & $254^{\mathrm{a}}$ & 6.5 & 4.5 \\
$\mathrm{~B} 1.2$ & 25.1 & 56 & 7.7 & 1.2 \\
$\mathrm{~B} 2.2$ & 28.5 & 230 & 6.8 & 4.3 \\
$\mathrm{C} 1.1$ & 35.1 & $40^{\mathrm{a}}$ & 19.6 & 2.2 \\
C2.1 & 37.4 & 344 & 16.3 & 15.4 \\
C3.1 & 32.2 & 414 & 19.4 & 22.0 \\
C4.1 & 34.9 & 414 & 15.5 & 17.5 \\
C1 & 38.7 & 230 & 20.5 & 13.0 \\
\hline
\end{tabular}

${ }^{\mathrm{a}}$ Maximum test duration until failure

the optical power increased further by $20 \%$ (initial power after 2nd IC $P_{\mathrm{I}}=211 \mu \mathrm{W}$ ). Laser B1.1 gradually degraded within 174 and failed after the 1 st IC. The replacement laser B1.2 was operated for 56 days under vacuum and showed a reduction of its optical power of $4 \%$. The optical power of the laser B3.1 decreased over the whole test duration and reduced severely within the last test month. On the 403th day its output power reached values below the defined laser 
failure limit of $50 \%$ initial power and even reduced further to $60 \%$ initial power at the end of the test. Laser B4.1 also revealed a significant drop in laser power. The optical power dropped from its initial value of $P_{\mathrm{I}}=194 \mu \mathrm{W}$ below the failure criterion after 254 days of operation and reached its minimum of $95 \mu \mathrm{W}$, which is just below the failure criterion (total reduction of 49.5\%). Then the laser power increased again until the end of the test to $196 \mu \mathrm{W}$, which is even above the initial value.

Since lasers B4.1 and B2.2 showed a similar laser power variation as laser B3.1, it could be possible that also the optical power of laser B3.1 recovered again but as the test was not continued further, there is no way of telling.

In Fig. $2 \mathrm{e}$ and $\mathrm{f}$ the behaviour of the optical power of lasers from supplier $\mathrm{C}$ are outlined. All lasers of supplier $\mathrm{C}$ had in common that the optical power started to increase after the beginning of the endurance test (2-7\% of $P_{\mathrm{I}}$ ) before it stabilized after several days. Therefore, an additional burnin would help to improve the stability of the optical output power.

Laser C1.1 showed proper function until it suddenly failed after 40 days. The failed $\mathrm{C} 1.1$ laser was replaced during the 1st IC by laser C1.2 and was sent back to its manufacturer for further investigations.

The variations of the optical power for lasers C2.1 and C1.2 were within $1 \%$ of their initial power.

The noise of the polarization-independent optical power of lasers C3.1 and C4.1, which is not seen in the measurement of the polarization-dependent optical power, is assumed to be caused by a random misalignment of the laser beam with respect to the optical detector in connection with the repeatedly moved linear translation stage. First both lasers C3.1 and C4.1 showed the typical increase in optical power. Then the optical power of laser C3.1 gradually reduced to approximately its initial value and the optical power of laser $\mathrm{C} 4.1$ remained $3 \%$ above its initial value after the end of the test.

After the completion of the endurance test the equivalent operational lifetime $\tau_{\text {eop }}$ of the tested lasers was calculated with the mean acceleration factor $\mathrm{AF}_{\text {mean }}$ and the test duration $\Delta t_{\text {test }}$ according to

$\tau_{\text {eop }}=\mathrm{AF}_{\text {mean }} \cdot \Delta t_{\text {test }}$

For the calculation of the mean acceleration value $\mathrm{AF}_{\text {mean }}$, first the acceleration factor of each data point $\mathrm{AF}_{i}$ according to Eq. (1) was calculated and then averaged.

In Table 2 the maximum equivalent operational life time $\tau_{\text {eop }}$ of each VCSEL is stated which would be reached based on the measurements at the end of this endurance test. Most of the lasers were still working within the defined pass/fail criterion ( $>50 \%$ of initial output power $P_{\mathrm{I}}$ ). For those lasers which failed during the test, the maximum reached lifetime was calculated up to the time when they failed.
The maximum equivalent operational lifetime $\tau_{\text {eop }}$ in Table 2 mainly varies for each VCSEL due to the different operational temperatures $T_{\text {op }}$ required to reach the defined laser frequency at the defined operational current $I_{\mathrm{op}}$. Thus, also the maximum equivalent operational lifetime is different for each laser.

\subsection{Test results from electro-optical characterisations}

In this section the results from the electro-optical characterisations are discussed. No results for lasers A2.1, B2.1 and C1.1 are presented in this section, since they have only been characterized once due to their failure prior to the first intermediate characterisation point and, therefore, no information on their aging behaviour could be obtained. The lasers B3.1 and B4.1 showed a reduction of their optical power of more than $50 \%$ but were still working at the time of the electrooptical characterisation and, therefore, they were also tested. Additionally, lasers C3.1 and C4.1 are not mentioned in the discussion, since a mishandling of the lasers after the endurance test caused a failure of the lasers.

In Fig. 4 an example of the measured laser current and laser temperature combinations to set the wavelength of the ${ }^{87} \mathrm{Rb}\left(5^{2} \mathrm{~S}_{1 / 2} F=2 \rightarrow 5^{2} \mathrm{P}_{1 / 2} F=1\right)$ transition as well as in the measurements of the current tuning coefficient are shown for laser A2.2. Both Fig. 4a and b contain the measurement results from the preliminary $\left(\tau_{\text {eop }}=0\right.$ a), intermediate $\left(\tau_{\text {eop }}=9.9\right.$ a) and the final characterisation $\left(\tau_{\text {eop }}=13.1\right.$ a) of laser A2.2.

In Fig. 4a the laser temperature $T_{\mathrm{op}}$ of laser A2.2 showed in total a reduction of $\Delta T_{\mathrm{op}}=(-3.1 \pm 0.2){ }^{\circ} \mathrm{C}$ between the preliminary and the final characterisation. Also the current tuning coefficient changed for the aged laser by $(24 \pm 16) \%$ (see Fig. 4b). Similar measurements have been carried out for all tested lasers. The change detected in the operational laser temperature $T_{\mathrm{op}}$ at $I_{\mathrm{op}}$ and the current tuning coefficient are summarized in Table 5 in the Appendix. The change of $T_{\mathrm{op}}$ at $I_{\mathrm{op}}$ for all lasers is additionally shown in Fig. 5.

Since each laser experienced a different aging in the experiment, the change of $T_{\mathrm{op}}$ over the equivalent operational lifetime $\tau_{\text {eop }}$ is depicted.

All lasers from supplier A (Fig. 5a) showed a reduction of $T_{\text {op }}$ over the test duration. The laser temperature $T_{\mathrm{op}}$ of laser A3.1 shifted by $(-6.1 \pm 0.2){ }^{\circ} \mathrm{C}$. Lasers from supplier B showed no clear trend (Fig. 5b). The laser temperature $T_{\mathrm{op}}$ of laser B4.1 as well as of laser B2.2 increased during the test but laser B1.1, which failed after the first intermediate characterisation, decreased.

Both tested lasers from supplier $\mathrm{C}$ showed an increase of $T_{\mathrm{op}}$. 

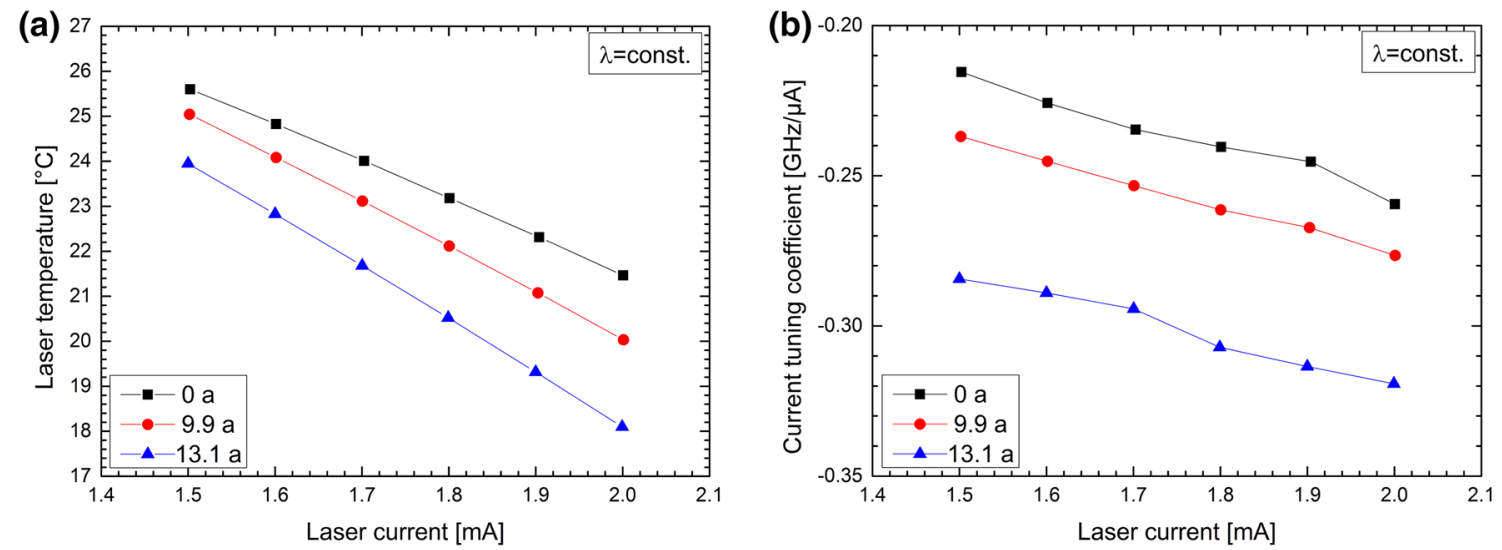

Fig. 4 Laser A2.2: a measurements of the laser current and laser temperature combinations to set the defined wavelength, $\mathbf{b}$ measurements of the current tuning coefficient

Fig. 5 Change of the operational laser temperature $T_{\mathrm{op}}$ at $I_{\text {op }}$ over the calculated equivalent operational lifetime: a lasers of supplier A, b lasers of supplier B and $\mathbf{c}$ lasers of supplier C

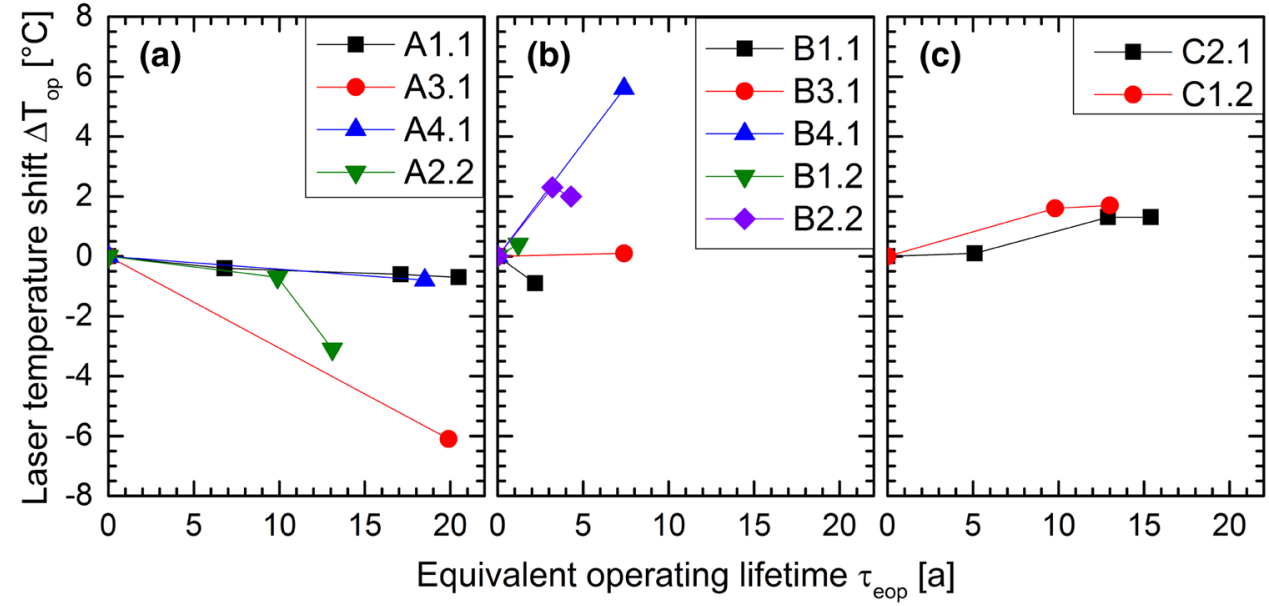

characterization. More lasers failed in vacuum chamber 1 than in vacuum chamber 2 . However, all lasers in vacuum chamber 1 and 2 were handled in the same way and powered from identical equipment. There was no known difference between the two vacuum chambers.

Lasers A2.1 and B2.1 failed shortly after test start and laser B1.1 also showed a clear reduction of its optical power right from the start. Most likely these lasers were already damaged at the test start.

However, one laser (C1.1) suddenly failed after 40 days of operation in vacuum chamber 1 . This laser was sent back to its manufacturer for further tests on the failure.

Investigations by the manufacturer indicated that this laser showed typical features of a laser suffered from an ESD event. Since the high sensitivity of VCSELs on ESD was known, several ESD precautions during handling and operation were made but due to the repeated handling and mounting of the lasers during the test, the risk of ESD damage cannot be eliminated completely. Therefore, the intermediate characterizations with the complete dismounting of 
the lasers entailed additional risks for ESD damage. Most of the lasers showed only a slight increase or reduction of their optical power after remounting which was explained by a different reassembly. However, a drop in the optical power, which for example laser A2.2 showed after the 2nd IC, could also be caused by a prior ESD event.

Due to the failure of the tested lasers, we decided to include measurements of the reverse current-voltage, forward current-voltage as well as the optical power-current characteristics in future test procedures. These additional measurements can help to detect defect or already degraded VCSELs before mounting them in a test setup. Especially, in the reverse current-voltage measurement a degradation, for example caused by an ESD event, can be identified by a flattening of the originally sharp breakdown voltage [17].

Two lasers from supplier A revealed polarization jumps (A3.1, A4.1). For applications which use polarization optics or a polarization-maintaining fibre, the preservation of the polarization plane is critical. In such applications polarization jumps could cause severe problems and result in a complete loss of the optical power. This was also one reason why in the CDSM application a multimode fibre, which connects the laser unit with the sensor unit, in combination with polarization optics at the sensor unit was chosen. After the multimode fibre the optical light is nearly unpolarised and the defined polarization state is obtained with the polarization optics in the sensor unit. Therefore, optical power is lost at the sensor unit but a failure caused by polarization jumps is avoided. However, the measurements have shown that the change of the polarization state could last up to $20 \mathrm{~h}$. Further investigation would be required to study this transition area and to evaluate the influence on the operation of the magnetometer. Additionally, depending on the rate of polarization jumps they could influence the CPT resonance shape $[18,19]$.

With respect to the JUICE mission it was important to test if a VCSEL laser can operate up to 17 years under vacuum condition. The endurance test allowed us to prove that lasers from at least supplier A and C can reach lifetimes of more than 17 years and still have enough optical power for the operation of the CDSM magnetometer. Lasers A1.1, A3.1 and A4.1 from supplier A as well as lasers C3.1 and C4.1 from supplier $\mathrm{C}$ reached an equivalent operational lifetime of more than 17 years. The equivalent operational lifetime for lasers of supplier B was much lower than the lifetime of the two other suppliers $\mathrm{A}$ and $\mathrm{C}$. This was due to the fact that the activation energy $E_{\mathrm{A}}$, which we had received from the manufacturer $\mathrm{B}$, is about half the value of the two other manufacturers. Thus, the calculated equivalent operational lifetime is much lower for lasers of supplier B.

The measurement on the laser temperature $T_{\mathrm{op}}$ at the operational laser current $I_{\mathrm{op}}$ for the required nominal wavelength of $\lambda=794.979 \mathrm{~nm}$ (vacuum wavelength) showed different trends for the three manufacturers (see Fig. 5). The laser temperature $T_{\mathrm{op}}$ for supplier A decreased for all tested lasers over time. Lasers from supplier B showed both reduction and increase of the laser temperature $T_{\mathrm{op}}$. The tested lasers from supplier $\mathrm{C}$ revealed an increase of their laser temperature $T_{\mathrm{op}}$ during the test campaign.

Laser A3.1 had the largest operational temperature shift of all tested lasers of $(-6.1 \pm 0.2){ }^{\circ} \mathrm{C}$ for an equivalent operational lifetime of about 20 years. The reason for this large change could be caused by a mode jump, which this laser showed several times during the vacuum test. Nevertheless, in the CDSM application such a change of the operational temperature $T_{\text {op }}$ can be compensated. However, for different applications enough margins in the design of the instrument should be taken into account.

Also laser B4.1, which failed due to a reduction of its initial power below the defined criterion, showed a large increase of $T_{\mathrm{op}}$ at $I_{\mathrm{op}}$ of $(+5.6 \pm 0.6){ }^{\circ} \mathrm{C}$. However, laser B3.1, which also showed a power reduction of $50 \%$ initial power, has no change of its operational temperature. All other lasers showed far less changes of $T_{\mathrm{op}}$ at $I_{\mathrm{op}}$.

The current tuning coefficient $\Delta \nu / \Delta I$ for the lasers of manufacturers $\mathrm{A}, \mathrm{B}$ and $\mathrm{C}$ also revealed differences. In general, lasers from supplier $\mathrm{C}$ showed the smallest current tuning coefficient and were a factor 2-3 smaller than for suppliers A and B (see Table 5). A smaller current tuning coefficient is a benefit for the operation of the magnetometer because laser current noise is less efficiently converted into frequency noise.

Most of the lasers showed no change of their current tuning coefficient within the measurement accuracy after aging. However, the current tuning value $\Delta \nu / \Delta I$ of lasers A3.1, A2.2, B1.1 and B3.1 increased (see Table 5; Fig. 4). The change of $\Delta \nu / \Delta I$, which is stated in column three of Table 3, was calculated between the initial characterization and the final characterization.

In the CDSM application an increase in the current tuning coefficient would cause an increase of the noise level and thus in a reduction of the signal-to-noise ratio.

The mean temperature tuning coefficient $\Delta \nu / \Delta T$ was identical for the lasers of the three suppliers (see Table 4).

Table 3 Change of the current tuning coefficient $\Delta \nu / \Delta I$ between initial and final electro-optical characterization: As was mentioned in Sect. 4.1 the lasers aged differently and thus they reached a different equivalent operational lifetime $\tau_{\text {eop }}$ at the end of the accelerated endurance test

\begin{tabular}{lcl}
\hline Laser & $\tau_{\text {eop }}$ (a) & Change of $\Delta \nu / \Delta I$ for $\tau_{\text {eop }}(\%)$ \\
\hline A3.1 & 19.9 & $30 \pm 23$ \\
A2.2 & 13.1 & $24 \pm 16$ \\
B1.1 & 2.2 & $11 \pm 8$ \\
B3.1 & 7.4 & $18 \pm 8$ \\
\hline
\end{tabular}


Table 4 Mean temperature tuning coefficient $\Delta \nu / \Delta T$ for the three different suppliers

\begin{tabular}{ll}
\hline Supplier & $\Delta \nu / \Delta T\left(\mathrm{GHz} /{ }^{\circ} \mathrm{C}\right)$ \\
\hline A & $-26.5 \pm 0.6$ \\
B & $-26.5 \pm 0.5$ \\
C & $-26.5 \pm 0.9$ \\
\hline
\end{tabular}

This parameter revealed a dependence neither on the laser current nor on the laser temperature and did not alter for increased operational lifetime.

The temperature tuning coefficient depends on the change of the average refractive index and the thermal expansion of the cavity with temperature [1]. The equality of the temperature tuning coefficients can possibly be explained by the fact that the material composition of the VCSELs is quite similar and thus they have almost the same linear temperature expansion coefficient. However, the current tuning coefficient seems to depend significantly on the exact inner structure of the lasers (e.g. size of the cavity, number of quantum wells, current confinement) and is thus unequal for the lasers from the three different suppliers.

VCSELs from supplier A showed typical threshold current values between 0.4 and $0.6 \mathrm{~mA}$. From supplier $\mathrm{C}$, the threshold current varied in the range from 1.3 to $1.5 \mathrm{~mA}$ and for supplier B from 0.7 to $0.8 \mathrm{~mA}$. Lasers from supplier A as well as lasers $C 2.1$ and $C 1.2$ showed no change of their threshold current over time within the measurement accuracy. On the other hand, lasers B1.1, B3.1 and B4.1 from supplier B revealed a clear increase of their threshold current over their operational lifetime. The threshold current of VCSEL B 1.1 and B3.1 increased from 0.8 to $1.3 \mathrm{~mA}$ and for laser B4.1 from 0.9 to $1.6 \mathrm{~mA}$. An increase of the threshold
Table 5 Summary of results obtained for the laser temperature $T_{\text {op }}$ and current tuning coefficient $\Delta \nu / \Delta I$ at $I_{\mathrm{op}}$

\begin{tabular}{|c|c|c|c|c|c|}
\hline Laser & $\Delta t_{\text {test }}$ (days) & $\tau_{\text {eop }}(\mathrm{a})$ & $T_{\mathrm{op}}\left({ }^{\circ} \mathrm{C}\right)$ & $\Delta T_{\mathrm{op}}\left({ }^{\circ} \mathrm{C}\right)$ & $\begin{array}{l}\Delta \nu / \Delta I(\mathrm{GHz} / \\
\mu \mathrm{A})\end{array}$ \\
\hline \multirow[t]{4}{*}{ A1.1 } & 0 & 0 & $24.2 \pm 0.1$ & & $-0.269 \pm 0.001$ \\
\hline & 114 & 6.8 & $23.8 \pm 0.1$ & $-0.4 \pm 0.2$ & $-0.29 \pm 0.04$ \\
\hline & 288 & 17.1 & $23.6 \pm 0.1$ & $-0.2 \pm 0.2$ & $-0.286 \pm 0.018$ \\
\hline & 344 & 20.5 & $23.5 \pm 0.1$ & $-0.1 \pm 0.2$ & $-0.29 \pm 0.03$ \\
\hline \multirow[t]{2}{*}{ A3.1 } & 0 & 0 & $26.5 \pm 0.1$ & & $-0.395 \pm 0.008$ \\
\hline & 414 & 19.9 & $20.4 \pm 0.1$ & $-6.1 \pm 0.2$ & $-0.51 \pm 0.09$ \\
\hline \multirow[t]{2}{*}{ A4.1 } & 0 & 0 & $27.3 \pm 0.1$ & & $-0.336 \pm 0.003$ \\
\hline & 414 & 18.5 & $26.5 \pm 0.1$ & $-0.8 \pm 0.2$ & $-0.38 \pm 0.05$ \\
\hline \multirow[t]{3}{*}{ A 2.2} & 0 & 0 & $22.4 \pm 0.1$ & & $-0.25 \pm 0.03$ \\
\hline & 174 & 9.9 & $21.7 \pm 0.1$ & $-0.7 \pm 0.2$ & $-0.270 \pm 0.010$ \\
\hline & 230 & 13.1 & $19.3 \pm 0.1$ & $-2.4 \pm 0.2$ & $-0.314 \pm 0.022$ \\
\hline \multirow[t]{2}{*}{ B1.1 } & 0 & 0 & $19.2 \pm 0.1^{\mathrm{a}}$ & & $-0.265 \pm 0.004$ \\
\hline & 114 & 2.2 & $18.3 \pm 0.3^{\mathrm{a}}$ & $-0.9 \pm 0.4$ & $-0.293 \pm 0.021$ \\
\hline \multirow[t]{2}{*}{ B3.1 } & 0 & 0 & $21.3 \pm 0.1$ & & $-0.313 \pm 0.011$ \\
\hline & 414 & 7.4 & $21.4 \pm 0.3^{\mathrm{a}}$ & $0.1 \pm 0.4$ & $-0.370 \pm 0.021$ \\
\hline \multirow[t]{2}{*}{ B4.1 } & 0 & 0 & $19.2 \pm 0.1$ & & $-0.313 \pm 0.011$ \\
\hline & 414 & 7.4 & $24.8 \pm 0.5^{\mathrm{a}}$ & $5.6 \pm 0.6$ & $-0.324 \pm 0.021$ \\
\hline \multirow[t]{3}{*}{ B1.2 } & 0 & 0 & $25.2 \pm 0.1$ & & $-0.292 \pm 0.022$ \\
\hline & 0 & 0 & $25.1 \pm 0.1$ & $-0.1 \pm 0.2$ & $-0.295 \pm 0.015$ \\
\hline & 56 & 1.2 & $25.5 \pm 0.1^{\mathrm{a}}$ & $0.4 \pm 0.2$ & $-0.295 \pm 0.013$ \\
\hline \multirow[t]{4}{*}{ B2.2 } & 0 & 0 & $28.5 \pm 0.1$ & & $-0.228 \pm 0.012$ \\
\hline & 0 & 0 & $28.3 \pm 0.2^{\mathrm{a}}$ & $-0.2 \pm 0.2$ & $-0.227 \pm 0.020$ \\
\hline & 174 & 3.2 & $30.6 \pm 0.1$ & $2.3 \pm 0.2$ & $-0.227 \pm 0.011$ \\
\hline & 230 & 4.3 & $30.3 \pm 0.2^{\mathrm{a}}$ & $-0.3 \pm 0.2$ & $-0.235 \pm 0.015$ \\
\hline \multirow[t]{4}{*}{$\mathrm{C} 2.1$} & 0 & 0 & $37.4 \pm 0.1$ & & $-0.115 \pm 0.009$ \\
\hline & 114 & 5.1 & $37.5 \pm 0.1$ & $0.1 \pm 0.2$ & $-0.115 \pm 0.008$ \\
\hline & 288 & 12.9 & $38.7 \pm 0.1$ & $1.2 \pm 0.2$ & $-0.113 \pm 0.005$ \\
\hline & 344 & 15.4 & $38.7 \pm 0.1$ & $0.0 \pm 0.2$ & $-0.112 \pm 0.005$ \\
\hline \multirow[t]{3}{*}{$\mathrm{C} 1.2$} & 0 & 0 & $38.7 \pm 0.1$ & & $-0.124 \pm 0.004$ \\
\hline & 174 & 9.8 & $40.3 \pm 0.1$ & $1.6 \pm 0.2$ & $-0.12 \pm 0.04$ \\
\hline & 230 & 13.0 & $40.2 \pm 0.1$ & $0.1 \pm 0.2$ & $-0.120 \pm 0.003$ \\
\hline
\end{tabular}

${ }^{\mathrm{a}}$ Values were determined by polynomial fit 
current can either be a typical result of an aged laser [1] or by ESD-driven degradation [20]. Either way it is a hint that the laser structure is already degraded to a certain degree.

Lasers from suppliers A and B had a typical line width of about $100 \mathrm{MHz}$ and VCSELs from supplier C about $50 \mathrm{MHz}$. A smaller laser line width is preferable for the CPT resonance excitation (see Sect. 2). The repeated measurement of the laser line width showed that the theoretical accuracy of $\pm 5 \mathrm{MHz}$ was not reached. Due to the required readjustment of the optical path for this measurement, an experimental accuracy of about $\pm 10 \mathrm{MHz}$ is obtained. Most of the laser line widths showed no significant change for aged lasers except for lasers B1.2, B2.2 and A4.1 which laser line widths' seemed to narrow. The narrowing could also be caused by a back reflection from an optical element into the laser.

The polarization ellipse was evaluated by measuring its maximum and minimum. The ratio of the maximum to minimum was used to evaluate changes. However, the accuracy of the measurement of the minimum was influenced by background light and, therefore, here an accuracy of $\pm 2 \mu \mathrm{W}$ was obtained. Within this limits no significant changes of the ratio were measured during the electro-optical characterisations.

Based on the results from the endurance test and the electro-optical characterizations we decided to further test lasers from supplier $\mathrm{C}$ for the CDSM prototype. Both lasers from suppliers $\mathrm{A}$ and $\mathrm{C}$ performed well during the endurance test. However, lasers from supplier $\mathrm{C}$ showed several benefits for the CDSM application compared to the other suppliers. They had no polarization jumps, less changes in the electro-optical parameters compared to the two other suppliers while aging, a lower current tuning coefficient, typically a two times smaller laser line width and a two to three times higher optical power than lasers from supplier A. The obtained output power from laser A was limited by the maximum allowed operational laser current $\left(I_{\max }=2 \mathrm{~mA}\right)$ according to the data sheet.

An endurance test with a bigger lot of lasers from supplier $\mathrm{C}$ will be performed within a new laser vacuum test facility at Graz University of Technology. The benefit of this new test facility is that it allows measurements of the electro-optical characteristic without dismounting the lasers. Additionally, the test facility allows the performance of a further burn-in to stabilize the optical parameters.

\section{Conclusion}

In summary, 16 VCSEL were tested under accelerated operational conditions under vacuum. Half of the VCSELs were operated for 344 days with two intermediate electro-optical characterizations (vacuum chamber 1) and the other half for 414 days without interference (vacuum chamber 2).

The majority of VCSELs did function well until the end of the test. The optical output power of VCSELs from suppliers $\mathrm{A}$ and $\mathrm{C}$ behaved stable with slight changes during the vacuum test $(<10 \%)$ except laser A3.1 which showed a decrease of $20 \%$. Lasers from supplier B showed the greatest variation in optical power during the accelerated aging test (up to 50\%). Such strong variations could influence the performance of our magnetometer. Four lasers in vacuum chamber 1 failed completely (A2.1, B2.1, C1.1 and B1.1).

With the endurance test we were able to show that VCSELs from supplier $C$ can survive more than 17 years of operation with no major degradation of their performance and thus they are well suited for long-term space missions like JUICE. However, a burn-in appeared to be needed for the selected supplier $\mathrm{C}$ to stabilize the optical power.

For the JUICE application we decided to further test lasers from supplier $\mathrm{C}$ and perform an endurance test with a bigger lot in a new laser vacuum test facility at Graz University of Technology. The benefit of this new test facility is that it allows measurements of the electro-optical characteristic in the endurance test setup without dismounting the lasers.

Acknowledgements Open Access Funding provided by Graz University of Technology. The support and expertise of the team at the Optics and Opto-Electronics Laboratories of the European Space Research and Technology Centre are greatly appreciated.

Funding The Austrian part of the development, operation and data evaluation was financially supported by the European Space Agency in the frame of a Strategic Initiative for Austrian (Contract 4000105726) as well as by rolling grants of the Austrian Academy of Sciences and the Graz University of Technology.

Open Access This article is distributed under the terms of the Creative Commons Attribution 4.0 International License (http://creativecommons.org/licenses/by/4.0/), which permits unrestricted use, distribution, and reproduction in any medium, provided you give appropriate credit to the original author(s) and the source, provide a link to the Creative Commons license, and indicate if changes were made.

\section{Appendix}

In Table 5 the results of the laser temperature $T_{\mathrm{op}}$ at the operational current $I_{\mathrm{op}}$ and the current tuning coefficients $\Delta \nu / \Delta I$ at $I_{\text {op }}$ are summarized.

With the help of the mean acceleration factor $\mathrm{AF}_{\text {mean }}$ from Table 2 and the test duration $\Delta t_{\text {test }}$ until the characterisation, which is stated in column two of Table 5, the equivalent operational lifetime $\tau_{\text {eop }}$ for each laser at the time of the characterisation was calculated and is stated in column three of Table 5. 
In the fourth column the measured laser temperature $T_{\text {op }}$ at the operational laser current $I_{\mathrm{op}}$ for the required nominal wavelength is listed. During the second and fourth electrooptical characterisation, the temperature at the operational laser current was not exactly measured at the defined laser current for lasers of company B. In that case the laser temperature was calculated from a second-order polynomial fit to the measured values [21]. In Table 5 those values are marked with the superscript a.

The temperature change $\Delta T_{\text {op }}$ between consecutive characterisations is given in the fifth column. In the sixth column the calculated current tuning coefficients $\Delta \nu / \Delta I$ for the operational laser current $I_{\text {op }}$ are summarized. The current tuning coefficients were calculated for $I_{\text {op }}$ using a linear fit to the measured values.

\section{References}

1. H.E. Li, K. Iga, Vertical-Cavity Surface-Emitting Laser Devices (Springer, Berlin, 2003)

2. C. Affolderbach, A. Nagel, S. Knappe, C. Jung, D. Wiedenmann, R. Wynands, Appl. Phys. B 70, 407 (2000)

3. R. Lammegger, Patent WO 2008/151344 A3 (2008)

4. J. Piris, L. Ferreira, B. Sarti, in Proceedings of the 12th European Conference on Spacecraft Structures, Materials and Environmental Testing (2012), p. 30

5. J. Piris, E.M. Murphy, M. Levy, G. Klumel, R. Diamant, B. Sarti, in Proc. SPIE 10563, International Conference on Space Optics - ICSO 2014 (2017), p. 105633A
6. A. Pollinger, Dissertation, Graz University of Technology, 2013

7. E. Arimondo, Prog. Opt. 35, 257 (1996)

8. R. Wynands, A. Nagel, Appl. Phys. B 68, 1 (1999)

9. G. Breit, I.I. Rabi, Phys. Rev 38, 2082 (1931)

10. D.A. Steck, Rubidium 87 D Line Data. http://steck.us/alkalidata (revision 2.1.5, 13 January 2015)

11. E. Breschi, G. Kazakov, R. Lammegger, G. Mileti, B. Matisov, L. Windholz, Phys. Rev. A 79, 063837 (2009)

12. E. Breschi, G. Kazakov, R. Lammegger, B. Matisov, L. Windholz, G. Mileti, IEEE Trans. Ultrason. Ferroelectr. Freq. Control 56, 926 (2009)

13. C. Hagen, M. Ellmeier, J. Piris, R. Lammegger, I. Jernej, W. Magnes, E. Murphy, A. Pollinger, C. Erd, W. Baumjohann, in Proc. SPIE 10563, International Conference on Space Optics ICSO 2014 (2017), p. 105634Y

14. R. Herrick, Jpn. J. Appl. Phys 51, 11PC01 (2012)

15. B.M. Hawkins, in 52nd Electronic Components and Technology Conference 2002 (2002), p. 540

16. H.-D. Kim, W.-G. Jeong, H.-E. Shin, J.-H. Ser, H.-K. Shin, Y.-G. Ju, Opt. Express 14, 12432 (2006)

17. O. Ueda, S.J. Pearton, Materials and Reliability Handbook for Semiconductor Optical and Electron Devices (Springer, New York, 2013)

18. J. Camparo, M. Huang, T. Driskell, in Joint European Frequency and Time Forum and International Frequency Control Symposium (2013), p. 612

19. M. Huang, J. Camparo, in Joint Conference of the IEEE International Frequency Control and the European Frequency and Time Forum Proceedings (2011), p. 1

20. T. Kim, T. Kim, S. Kim, S.B. Kim, ETRI J. 30, 833 (2008)

21. A. Lytkine, Spectrochim. Acta Part A Mol. Biomol. Spectrosc. 63, 940 (2006) 